\title{
Bovins ensauvagés et bovins domestiques dans le désert de Chihuahua (Mexique) : suivi radiotélémetrique et résultats
}

\author{
L Hernández ${ }^{1}$, H Barral 2 \\ 1/nstituto de Ecologia, AC Apdo Postal 632, 34100 Durango, Dgo Mexique ; ${ }^{O}$ ORSTOM, \\ Département MAA, BP 5045, 34032 Montpellier, Cedex 1, France
}

\begin{abstract}
Au Mexique, 2 facteurs favorisent l'ensauvagement du bétail : la grande extension des propriétés et la faiblesse des investissements en matière d'infrastructures (clôtures, points d'eau aménagés) dans les zones d'élevage extensif.
\end{abstract}

L'importance des études portant sur ce thème dans le désert "chihuahuense" s'explique par le fait que les bovins "ensauvagés" y coexistent généralement avec le bétail domestique, et qu'il existe donc un risque de concurrence, entre ces deux types de bétail pour l'utilisation des ressources fourragères, au détriment à la fois de l'écosystème et du bétail domestique.

Ce travail a été effectué dans la région sud-est de la Réserve de la Biosphère de Mapimí, dans une aire de 115000 ha sans clôtures, à l'intérieur de laquelle ont été décrites 10 formations végétales (Montaña, 1988, Instituto de Ecología, AC México, 167-197) et où il existe environ 6000 têtes de bétail dont 1000 têtes de bovins ensauvagés. Pour déterminer les fréquences d'utilisation de ces 10 formations végétales, il a été fait appel aux techniques de radiotélémétrie, en fixant des colliers équipés d'émetteurs-radio à 3 vaches ensauvagées et à 3 vaches domestiques qui ont été suivies pendant des cycles de 24 heures durant une année. Les localisations obtenues ont été ensuite reportées sur une carte des formations végétales à l'échelle du 1/50 000. Enfin, l'on a calculé les surfaces d'aires d'activité de chaque individu au moyen de la méthode du plus petit polygone convexe.
Grâce aux 693 et 439 localisations par radio correspondant respectivement aux vaches ensauvagées et aux vaches domestiques, nous avons obtenu 2 résultats bien établis en matière d'utilisation de l'espace concernant ces 2 types de bétail :

1. Les vaches ensauvagées ont des aires d'activité très supérieures à celles des vaches domestiques ( $47 \mathrm{~km}^{2}$ contre $\left.14 \mathrm{~km}^{2}\right)$.

2. Les vaches ensauvagées tirent parti de milieux beaucoup plus diversifiés (hauts glacis, zones planes et lits de cours d'eau à écoulement temporaire), ce qui se traduit par un meilleur usage différentiel des ressources alimentaires, dans des zones dont la production est comprise entre 1000 et $1500 \mathrm{~kg}$ de matière sèche par hectare et par an. Les vaches domestiques, en revanche, se concentrent dans des zones d'écoulements saisonniers qui correspondent en fait à des axes d'érosion hydrique à proximité des points d'eau, avec une production comprise entre 500 et $1000 \mathrm{~kg}$ de matière sèche par hectare et par an.

Pour conclure, il existe dans la Réserve de la Biosphère de Mapimí des différences bien marquées en matière d'utilisation de l'espace entre bovin domestique et bovin ensauvagé, ce qui explique qu'aient pu se maintenir de manière séparée ces 2 populations. Enfin, l'utilisation quasi-permanente d'un seul type de formation végétale par le bétail domestique est la marque d'une mauvaise gestion des parcours, de nature à provoquer des dégâts écologiques. 